\title{
Concentraciones sanguíneas de ß-hidroxibutirato, NEFA, colesterol y urea en cabras lecheras de tres rebaños con sistemas intensivos de producción y su relación con el balance nutricional
}

\author{
Relationship between blood metabolites ( $\beta$-hydroxybutirate, NEFA, cholesterol and urea) and \\ nutritional balance in three dairy goat herds under confinement \\ C. Ríos ${ }^{1}$, M P Marín'1, M Catafau', F Wittwer² \\ ${ }^{1}$ Escuela de Medicina Veterinaria, Universidad Santo Tomás. Av. Ejército 146, Santiago, Chile. \\ ${ }^{2}$ Instituto de Ciencias Clínicas Veterinarias, Universidad Austral de Chile, Valdivia, Chile.
}

\section{SUMMARY}

\begin{abstract}
The aim of this study was to assess some blood metabolites as indicators of nutritional balance in dairy goats under intensive conditions. Three dairy herds were selected and 21 goats chosen in each. Goats were separated into three groups of seven animals each according to their productive stage: pre-partum (PP), 3-6 weeks of lactation (T1) and 16-20 weeks of lactation (T2). Blood samples were obtained during the first milking of the day and $\beta$ - hydroxybutirate (BHB), non-esterified fatty acids (NEFA), cholesterol, and urea were determined by colorimetric methods. Body condition score (BCS) and milk production were also registered. To establish protein (g/day) and metabolic energy (Mcal/day) content of the ration fed, a proximal chemical analysis was performed. Nutrient requirements of goats were determined by NRC. In the three herds, significant high values of NEFA $(\mathrm{P}<0.05)$ were coincident with a non -significant increase in milk production $(\mathrm{P}<0.05)$ and in one herd with a decrease in $\mathrm{BCS}(\mathrm{P}<0.05)$, which suggests fat mobilization. Urea concentration was between $10.0 \pm 1.5$ and $16.2 \pm 1.5 \mathrm{mmol} / \mathrm{L}$, higher than that reported by other authors. Every herd and productive stage presented imbalances between the protein and energy contents of the ration supplied, with high protein and low energy intake, which was shown in the blood metabolites analyzed. Accordingly, selected blood metabolites allowed the identification of nutritional imbalances in the studied herds.
\end{abstract}

Palabras clave: cabras, metabolitos sanguíneos, nutrición.

Key words: goats, blood metabolites, nutrition.

\section{INTRODUCCION}

La explotación caprina en Chile ha experimentado un auge en los últimos años, desarrollándose empresas con altos niveles de intensificación en sus procesos productivos, que persiguen principalmente obtener el máximo en producción de leche con un mínimo de costos. Es claro que cuanto más alto sea el potencial productivo del rebaño, mayor será el riesgo de provocar trastornos metabólicos en los animales, producto del desbalance entre el ingreso y egreso de nutrientes al organismo. En ganado bovino, estas alteraciones han sido ampliamente estudiadas, desarrollándose los Perfiles Metabólicos como una herramienta para el estudio y diagnóstico de desbalances nutricionales, mediante la determinación de metabolitos sanguíneos indicadores de las vías metabólicas de proteínas, energía y minerales (Wittwer 2000). En caprinos, también se ha utilizado el análisis de metabolitos sanguíneos

Aceptado: 12.07.2005 y de otros fluidos orgánicos, como leche, para evaluar el balance nutricional de la cabra lechera (Cabiddu y col 1998; Khaled y col 1999). En esta especie, se han descrito como indicadores del aporte nutricional las determinaciones de las concentraciones sanguíneas de glucosa, $\beta$-hidroxibutirato $(\beta \mathrm{HB})$, cuerpos cetónicos totales, ácidos grasos no esterificados (NEFA), colesterol, triglicéridos y urea (Amer y col 1999; Khaled y col, 1999, Ríos y col 2001, Khaled e Illek 2002). Por otro lado, ciertos indicadores productivos como producción de leche y condición corporal complementan los indicadores sanguíneos, pues también se ven afectados por los desbalances anteriormente mencionados (Cabiddu y col 1998).

Basado en la información señalada, por medio del presente trabajo se entregan antecedentes sobre las concentraciones sanguíneas de $\beta$-hidroxibutirato, NEFA, colesterol y urea como indicadores del balance metabólico de energía y proteínas en cabras lecheras y se analizan, junto con la condición corporal, producción de leche y su eventual asociación con el aporte de energía y proteína de la ración. 


\section{MATERIAL Y METODOS}

Se trabajó con tres rebaños caprinos lecheros de raza Saanen y mestiza Saanen ubicados en la Región Metropolitana de Santiago, Chile. Los animales eran mantenidos en confinamiento permanente, donde recibían una dieta basada en forraje (alfalfa fresca, heno y silo de maíz), concentrado comercial y subproductos de frutas (orujo, cáscara de almendra, desecho de pasa) en proporción promedio de 60,10 y $30 \%$ respectivamente.

En cada plantel se escogieron 21 cabras de dos o más lactancias, sometidas a dos ordeñas mecánicas diarias en los rebaños A y B y tres el rebaño C, con promedios de producción que fluctuaron entre 2 y 3,3 litros/día, las que se distribuyeron en los siguientes grupos:

Siete animales a finales de gestación, correspondientes al grupo preparto (PP); siete entre la $3^{\mathrm{a}}$ y $6^{\mathrm{a}}$ semana de lactancia, correspondientes al primer tercio de lactancia (T1); siete entre las 16 y 20 semanas de lactancia, correspondientes al segundo tercio de lactancia (T2). A los animales seleccionados se les registró la producción promedio de leche (L/día) y condición corporal (CC), esta última mediante escala visual ( 1 a 5 ) según el método descrito por Chunleau (1994).

Durante el período de mayo a noviembre del año 2000 se obtuvieron muestras de sangre durante la primera ordeña del día de los animales seleccionados mediante venopunción yugular, utilizando tubos al vacío sin anticoagulante. Estas fueron transportadas a temperatura de refrigeración al Laboratorio de Patología Clínica de la Universidad Santo Tomás, donde fueron centrifugadas para la obtención de suero (3.000 rpm x $10 \mathrm{~min}$ ), el que fue congelado $\left(-20^{\circ} \mathrm{C}\right)$ para su posterior análisis en serie al final del período de recolección.

Para cada etapa de lactancia analizada se recolectaron muestras de los insumos que componían las distintas raciones y se estimó el consumo diario de alimento por animal (kg/día), basándose en la fórmula de cálculo de capacidad de consumo entregada por ITOVIC (1982).

Análisis de laboratorio: En las muestras de suero se efectuó la determinación de las concentraciones de: $\beta$-hidroxibutirato ( $\beta \mathrm{HB}$ ) mediante método UV enzimático NAD dependiente (Randox, art. 1007), ácidos grasos no esterificados (NEFA) mediante método enzimático colorimétrico (Randox, art. 115), colesterol (COL) mediante el método colesterol-oxidasa (Stambio, art. 1010) y urea mediante el método ureasa/NADH (Roche, art. 1360604). Las muestras se procesaron en un analizador bioquímico semiautomático Microlab 200 (Merck).

Para determinar la composición nutritiva de la ración se utilizó el método convencional de Análisis Químico Proximal (AQP), donde se determinaron los porcentajes de materia seca (MS), extracto etéreo (EE), proteína cruda (PC, macro Kjeldahl), cenizas, fibra cruda (FC) y extracto no nitrogenado (ENN), según los métodos conven- cionales de la AOAC (1984). Para obtener el aporte de Energía Metabolizable (EM, Kcal/g alimento) de la ración se utilizó la ecuación de predicción para forrajes y concentrados a partir de la composición de la materia seca (IICA, 1992).

Para el cálculo de los requerimientos de EM (Mcal/ día) y Proteína Total (PT, g/día) de mantención y producción se utilizó la tabla de requerimientos descrita para cabras por el NRC (1981), considerando un porcentaje de grasa láctea de $3,5 \%$.

Se estimaron las diferencias porcentuales entre aportes y requerimientos energético-proteicos de las raciones suministradas y su posible relación con las variables sanguíneas analizadas.

Las variables sanguíneas fueron analizadas individualmente y descritas mediante promedio y desviación estándar (DE). La comparación de los valores entre las etapas productivas (PP, T1, T2) dentro de cada rebaño se realizó mediante análisis de varianza (ANDEVA), con un $95 \%$ de confianza, identificando las diferencias entre etapas productivas mediante la Prueba de Tukey. En los casos en que la distribución de los datos no fue normal, se utilizó la Prueba de Kruskall-Wallis $(\mathrm{P}<0,05)$. Para lo anterior se utilizó el paquete estadístico S.A.S/STAT (1985).

\section{RESULTADOS}

Los valores promedio y desviación estándar (DE) de las variables productivas (producción de leche, CC) y metabolitos sanguíneos analizados en los tres rebaños y según etapa de lactancia se muestran en el cuadro 1. Con respecto al producción de leche (PL), esta no presentó diferencias significativas $(\mathrm{P}>0,05)$ entre las etapas de producción en ninguno de los rebaños. A pesar de ello, se aprecian valores más altos de PL en T1 en los rebaños A y $\mathrm{C}$, los que coinciden con una disminución significativa de $\mathrm{CC}(\mathrm{P}<0,05)$ en el rebaño A. En el rebaño B se observa el mismo comportamiento de PL y CC en T2.

Los promedios de $\beta \mathrm{HB}$ fueron mayores en $\mathrm{T} 1$ en el rebaño A e igualmente altos en PP y T2 en el rebaño B. En el rebaño $C$ no se presentaron diferencias entre las distintas etapas de producción ( $\mathrm{P}>0,05)$.

En los rebaños A y $\mathrm{C}$ se presentó un aumento significativo de NEFA en T1, que en el rebaño A coincide con la disminución significativa de CC. En el caso del rebaño B, este aumento de NEFA se presenta en T2.

El colesterol no presentó diferencias entre las etapas analizadas en los rebaños A y B, existiendo un aumento significativo de su promedio en T2 en el rebaño C.

La concentración de urea presentó sus promedios más altos en PP y T2 en el rebaño A y en T1 y T2 en el rebaño $\mathrm{B}(\mathrm{P}<0,05)$. El rebaño $\mathrm{C}$ no presentó diferencias entre las etapas analizadas $(\mathrm{P}>0,05)$.

En el cuadro 2 se señalan los requerimientos de PT y EM y los aportes de la ración para los distintos rebaños y 
Cuadro 1. Promedios $( \pm \mathrm{DE})$ de producción de leche, condición corporal y de las concentraciones séricas de $\beta$ hidroxibutirato, NEFA, colesterol y urea en caprinos de tres rebaños determinados en los períodos de preparto (PP), inicio de lactancia (T1) y mediados de lactancia (T2).

Mean values $( \pm$ SD) of milk production, body condition score and blood metabolites in three dairy goat herds in three different productive stages (PP, T1, T2).

\begin{tabular}{|c|c|c|c|c|}
\hline Rebaño & Variable & PP & $\mathrm{T} 1$ & $\mathrm{~T} 2$ \\
\hline A & $\begin{array}{l}\text { Leche (L/día) } \\
\text { CC }(1 \text { a } 5) \\
\beta \text { HB }(\mathrm{mmol} / \mathrm{L}) \\
\text { NEFA }(\mathrm{mmol} / \mathrm{L}) \\
\text { COL }(\mathrm{mmol} / \mathrm{L}) \\
\text { UREA }(\mathrm{mmol} / \mathrm{L})\end{array}$ & $\begin{array}{c}3,4 \pm 0,3^{\mathrm{a}} \\
0,2 \pm 0,1^{\mathrm{a}} \\
0,4 \pm 0,2^{\mathrm{a}} \\
2,7 \pm 0,6 \\
11,3 \pm 2,0^{\mathrm{ab}}\end{array}$ & $\begin{array}{c}2,2 \pm 0,8 \\
2,8 \pm 0,4^{\mathrm{b}} \\
0,7 \pm 0,2^{\mathrm{b}} \\
2,5 \pm 0,9^{\mathrm{b}} \\
2,6 \pm 0,5 \\
10,0 \pm 1,5^{\mathrm{a}}\end{array}$ & $\begin{array}{c}2,1 \pm 0,6 \\
3,4 \pm 0,4^{\mathrm{a}} \\
0,2 \pm 0,2^{\mathrm{a}} \\
1,0 \pm 0,9^{\mathrm{a}} \\
2,5 \pm 0,6^{\mathrm{a}} \\
13,1 \pm 2,1^{\mathrm{b}}\end{array}$ \\
\hline B & $\begin{array}{l}\text { Leche (L/día) } \\
\text { CC }(1 \text { a } 5) \\
\beta H B(m m o l / L) \\
\text { NEFA }(\mathrm{mmol} / \mathrm{L}) \\
\text { COL }(\mathrm{mmol} / \mathrm{L}) \\
\text { UREA }(\mathrm{mmol} / \mathrm{L})\end{array}$ & $\begin{array}{c}3,0 \pm 0,4 \\
1,1 \pm 0,1^{\mathrm{a}} \\
0,4 \pm 0,2^{\mathrm{a}} \\
3,1 \pm 1,1 \\
11,3 \pm 1,7^{\mathrm{a}}\end{array}$ & $\begin{array}{c}1,9 \pm 0,2 \\
3,0 \pm 0,4 \\
0,4 \pm 0,4^{\mathrm{b}} \\
0,3 \pm 0,1^{\mathrm{a}} \\
2,6 \pm 1,2 \\
16,2 \pm 1,5^{\mathrm{b}}\end{array}$ & $\begin{array}{c}2,2 \pm 0,6 \\
2,9 \pm 0,4 \\
0,7 \pm 0,5^{\mathrm{ab}} \\
2,3 \pm 1,2^{\mathrm{b}} \\
3,7 \pm 0,8^{\mathrm{b}} \\
14,6 \pm 3,3^{\mathrm{b}}\end{array}$ \\
\hline $\mathrm{C}$ & $\begin{array}{l}\text { Leche (L/día) } \\
\text { CC }(1 \text { a } 5) \\
\beta \text { HB }(\mathrm{mmol} / \mathrm{L}) \\
\text { NEFA }(\mathrm{mmol} / \mathrm{L}) \\
\text { COL }(\mathrm{mmol} / \mathrm{L}) \\
\text { UREA }(\mathrm{mmol} / \mathrm{L})\end{array}$ & $\begin{array}{c}3,2 \pm 0,3 \\
0,3 \pm 0,4 \\
0,7 \pm 0,6^{\mathrm{a}} \\
1,7 \pm 0,6^{\mathrm{a}} \\
10,4 \pm 1,6\end{array}$ & $\begin{array}{l}3,6 \pm 0,9 \\
3,1 \pm 0,3 \\
0,3 \pm 0,2 \\
1,9 \pm 0,5^{\mathrm{b}} \\
2,6 \pm 0,8^{\mathrm{ab}} \\
13,0 \pm 1,9\end{array}$ & $\begin{array}{l}3,1 \pm 0,9 \\
3,2 \pm 0,3 \\
0,4 \pm 0,1 \\
1,4 \pm 0,3^{b} \\
3,3 \pm 0,6^{b} \\
12,3 \pm 2,1\end{array}$ \\
\hline
\end{tabular}

Letras diferentes en una misma fila indican diferencias significativas entre grupos $(\mathrm{P} \leq 0,05)$.

Cuadro 2. Requerimientos* y aportes de proteína total y energía metabolizable y su diferencia porcentual en los períodos de preparto (PP), inicio de lactancia (T1) y mediados de lactancia (T2) en caprinos de tres rebaños lecheros.

Protein and metabolizable energy requirements in three herds of dairy goats and its difference (\%) in prepartum (PP), early lactation (T1) and middle lactation (T2).

\begin{tabular}{ccccccccc}
\hline & & \multicolumn{3}{c}{ Proténa Total } & \multicolumn{3}{c}{ Energía Metabolizable } \\
Rebaño & $\begin{array}{c}\text { Etapa de } \\
\text { Producción }\end{array}$ & $\begin{array}{c}\text { Req. } \\
\text { (g/día) }\end{array}$ & $\begin{array}{c}\text { Aporte } \\
\text { (g/día) }\end{array}$ & $\begin{array}{c}\text { Dif. } \\
\%\end{array}$ & $\begin{array}{c}\text { Req. } \\
\text { (Mcal/día) }\end{array}$ & $\begin{array}{c}\text { Aporte } \\
\text { (Mcal/día) }\end{array}$ & $\%$ \\
\hline \multirow{4}{*}{ A } & PP & 138,3 & 338,3 & 69 & 4,49 & 2,99 & $(38)$ \\
& T1 & 270,3 & 409,1 & 37 & 5,83 & 4,63 & $(21)$ \\
& T2 & 273,44 & 342,6 & 25 & 5,99 & 4,5 & $(24)$ \\
& PP & 200,0 & 317,4 & 59 & 4,49 & 3,84 & $(14)$ \\
B & T1 & 249,2 & 408,9 & 64 & 5,44 & 4,51 & $(17)$ \\
& T2 & 278,2 & 473,7 & 70 & 6,07 & 4,82 & $(20)$ \\
& PP & 200 & 277,3 & 39 & 4,49 & 3,74 & $(17)$ \\
& C & T1 & 363,5 & 478,7 & 32 & 7,51 & 5,91 & $(21)$ \\
& T2 & 343,5 & 398,3 & 16 & 7,25 & 8,23 & 13 \\
\hline
\end{tabular}

* Requerimientos según NRC, 1981; ( )= valores negativos.

etapas estudiadas. Al respecto, se puede observar que para los tres rebaños en las tres etapas estudiadas existen aportes de PT por sobre los requerimientos de los animales que fluctúan entre $16 \%$ y $70 \%$. Por el contrario, los apor- tes de EM fueron insuficientes para cubrir los requerimientos entre un 14 y $38 \%$. La excepción la constituye el rebaño $\mathrm{C}$ en $\mathrm{T} 2$, que presentó un exceso de proteína y energía.

Los aportes energéticos y proteicos de la ración junto con los valores de los metabolitos sanguíneos obtenidos en el estudio se presentan en el cuadro 3. En él se aprecia que los altos valores de NEFA, $\beta \mathrm{HB}$ y urea coinciden con dietas con excesiva proteína y escasa energía.

Cuadro 3. Relación entre los aportes de proteína (P) y energía (E) con la concentración sérica de $\beta \mathrm{HB}$, NEFA, colesterol y urea en los períodos de preparto (PP), inicio de lactancia (T1) y mediados de lactancia (T2) en caprinos de tres rebaños lecheros.

Protein and energy intake and its relationship with serum $\beta$-hydroxybutirate $(\beta \mathrm{HB})$ in three dairy goat herds in three different production stages $(\mathrm{PP}, \mathrm{T} 1, \mathrm{~T} 2)$

\begin{tabular}{|c|c|c|c|c|c|c|}
\hline Rebaño & Grupo & $\begin{array}{l}\text { Aporte } \\
\text { P E }\end{array}$ & $\begin{array}{c}\beta \mathrm{HB} \\
\mathrm{mmol} / \mathrm{L}\end{array}$ & $\begin{array}{c}\text { NEFA } \\
\mathrm{mmol} / \mathrm{L}\end{array}$ & $\begin{array}{c}\mathrm{COL} \\
\mathrm{mmol} / \mathrm{L}\end{array}$ & $\begin{array}{c}\text { UREA } \\
\mathrm{mmol} / \mathrm{L}\end{array}$ \\
\hline \multirow[t]{3}{*}{ A } & PP & $\uparrow P \downarrow E$ & 0,65 & 0,40 & 2,7 & 11,3 \\
\hline & $\mathrm{T} 1$ & $\uparrow P \downarrow E$ & 0,15 & 2,52 & 2,6 & 10,0 \\
\hline & $\mathrm{T} 2$ & $\uparrow P \downarrow E$ & 0,16 & 0,97 & 2,5 & 13,1 \\
\hline \multirow[t]{3}{*}{ B } & PP & $\uparrow P \downarrow E$ & 0,8 & 0,41 & 3,0 & 11,3 \\
\hline & $\mathrm{T} 1$ & $\uparrow P \downarrow E$ & 0,39 & 0,30 & 3,1 & 16,3 \\
\hline & $\mathrm{T} 2$ & $\uparrow P \downarrow E$ & 0,7 & 2,27 & 3,7 & 14,6 \\
\hline \multirow[t]{3}{*}{$\mathrm{C}$} & PP & $\uparrow P \downarrow E$ & 0,31 & 0,67 & 1,7 & 10,4 \\
\hline & $\mathrm{T} 1$ & $\uparrow P \downarrow E$ & 0,31 & 1,94 & 2,6 & 13,0 \\
\hline & $\mathrm{T} 2$ & $\uparrow P \downarrow E$ & 0,40 & 1,35 & 3,3 & 12,3 \\
\hline
\end{tabular}

$\mathbf{P} \uparrow=$ Exceso de proteína con relación a los requerimientos.

$\mathbf{E} \uparrow=$ Exceso de energía con relación a los requerimientos.

$\mathbf{E} \downarrow=$ Déficit de energía con relación a los requerimientos.

\section{DISCUSION}

Al analizar la producción de leche se puede observar que los promedios de producción son similares a los descritos por Marín y col (2000) para cabras Saanen estabuladas de la zona central. Estos mismos autores describen los máximos de producción alrededor de la sexta semana de lactancia, lo que no se observó en este estudio, ya que no se presentaron diferencias significativas en las distintas etapas de producción. Con respecto a la CC, esta presentó una disminución significativa en el rebaño A en T1, lo que es atribuido principalmente a la movilización de reservas para sostener los niveles de producción en una etapa en que los requerimientos nutricionales son altos (Cabiddu y col 1998). En este mismo rebaño, la disminución de la CC coincide con un aumento significativo de NEFA, el que se asocia con un déficit en el aporte energético de la ración, lo que sumado a los altos requerimientos de la cabra en la primera etapa de lactancia o en el período de mayor producción lechera, provoca una movilización de reservas corporales con el consecuente au- 
mento de los ácidos grasos libres (Hussain y col 1996). Si bien en los rebaños $\mathrm{B}$ y $\mathrm{C}$ no se presentan variaciones de la $\mathrm{CC}$, existen aumentos significativos de NEFA en las diferentes etapas de producción, lo que puede ser atribuido al déficit de alrededor de un $20 \%$ en el aporte energético de la ración.

Con respecto al $\beta \mathrm{HB}$, el valor promedio de las $63 \mathrm{ca}-$ bras analizadas fue de $0,47 \mathrm{mmol} / \mathrm{L}$ con un rango de 0,01 a $1,26 \mathrm{mmol} / \mathrm{L}$. Este promedio coincide con el descrito por Chang y col (1997) para cabras en lactancia de 0,52 $\mathrm{mmol} / \mathrm{L}$. Los mayores valores encontrados en $\mathrm{T} 1$ en el rebaño A coinciden con el aumento de NEFA y disminución de $\mathrm{CC}$, lo que concuerda con el déficit energético presentado por estos animales, puesto que este es un cuerpo cetónico producto de la oxidación parcial de los ácidos grasos y su determinación se ha descrito como un indicador confiable de desbalance energético (Contreras y col 1996). Si bien algunos de los valores encontrados se podrían relacionar, según los autores antes mencionados, con la presentación clínica de cuadros de cetosis, estos no fueron pesquisados por estar más allá de los objetivos fijados para el presente estudio.

El comportamiento del colesterol no presentó diferencias entre las distintas etapas analizadas en los rebaños A y B, encontrándose valores promedio similares a los descritos por otros autores (Matthews 1999, Chiofalo y col 2000, Khaled e Illek 2000). En el rebaño C se vio un aumento significativo de esta variable en T2, coincidiendo con que esta fue la única etapa en que el aporte energético de la ración excedió a los requerimientos. Si bien este valor de colesterol no supera los rangos descritos como normales por los autores antes mencionados, Bertoni (1999) describe en bovinos lecheros un aumento del valor de colesterol asociado a dietas con un mayor contenido energético.

Los valores promedio de urea encontrados en el presente estudio fluctuaron entre los 10,05 y 16,25 mmol/L, superiores a los señalados en otros estudios, los que fluctuaron entre 7,2 y 8,8 mmol/L (Bed y col 1998, Khaled y col 1999, Tanwar y col 2000). Diversos autores han señalado que valores altos de urea se encuentran en rebaños que utilizan dietas con alto aporte proteico, describiendo también una estrecha relación entre la concentración de urea y la relación proteína-energía de la dieta de vacas, ovejas y cabras (Oltner y Wiktorsson 1983, Gustafsson y Carlson 1993, Wittwer y col 1993, Bed y col 1998, Cannas y col 1998). Lo anterior es debido a que tanto los aumentos de proteína dietaria como los déficit de energía determinan un aumento de la concentración de amonio ruminal y la urea se sintetiza en el hígado a partir del amonio, en cantidades proporcionales a la concentración ruminal de éste (Cunningham, 1996). En el cuadro 2 se puede observar que los aportes proteicos superan en los tres rebaños y en todas las etapas estudiadas los requerimientos de las cabras, fluctuando estos excesos entre un 16 y un $70 \%$. En el rebaño B, el promedio de urea de los animales estu- diados fue de 14,0 $\mathrm{mmol} / \mathrm{L}$, cercano al valor máximo del rango descrito en este estudio (cuadro 3), siendo éste el predio que presentó los mayores excesos en el aporte proteico (59-70\%). Una situación similar fue descrita por Ríos y col (2001), quienes encontraron altas concentraciones de urea tanto en sangre como en leche de cabras Saanen estabuladas, atribuyéndolas también a excesos de aporte proteico de la dieta.

Los cambios en los metabolitos sanguíneos descritos en este estudio concuerdan con Greppi y col (1995), quienes señalan que valores altos de NEFA, cuerpos cetónicos y urea en los primeros meses de lactancia en cabras lecheras se asocian con déficit energético e inducen cambios metabólicos considerables en cabras lecheras de alta producción.

Los resultados permiten concluir que los aumentos de metabolitos sanguíneos indicadores del balance energético (NEFA, $\beta \mathrm{HB}$ ) coinciden con un déficit en el aporte energético de la ración y los altos valores de urea encontrados pueden ser atribuidos a un exceso de aporte proteico y, en algunos casos, a déficit energético. Por lo tanto, los indicadores productivos y metabolitos sanguíneos analizados permitieron identificar la presencia de desbalances, de distintas magnitudes, entre aportes y requerimientos de energía y proteína en los rebaños caprinos lecheros analizados.

\section{RESUMEN}

El objetivo de este estudio fue determinar el comportamiento de algunos metabolitos sanguíneos indicadores del balance metabólico nutricional en la cabra lechera, sometida a sistemas intensivos de producción. Para ello se utilizaron tres rebaños de la región metropolitana de Chile, con 21 cabras cada uno. Los animales fueron divididos en grupos de 7 según su etapa de producción, siendo éstos preparto (PP), 3 a 6 semanas de lactancia (T1) y 16 a 20 semanas de lactancia (T2). Las determinaciones sanguíneas efectuadas correspondieron a $\beta$-hidroxibutirato $(\beta \mathrm{HB})$, ácidos grasos no esterificados (NEFA), colesterol y urea, todas realizadas mediante métodos colorimétricos. Además se registró producción de leche y condición corporal (CC) en forma individual. Para determinar el aporte de proteína total y energía metabolizable de la ración se realizó un análisis químico proximal de los insumos que componían la dieta de cada grupo productivo analizado. En los tres rebaños analizados se encontraron aumentos significativos de NEFA $(\mathrm{P}<0,05)$, los que se asociaron en un rebaño con disminución de la CC. Estos períodos coinciden con déficit en el aporte energético encontrado. Se observaron valores mayores de $\beta \mathrm{HB}$ que los descritos por otros autores, situación que también se explica por el escaso aporte energético de las raciones. Los valores promedio de urea fueron superiores a los reportados por otros autores, situación que se atribuye a un exceso de aporte proteico en las dietas.

En todos los rebaños, en los diferentes estados productivos, se encontraron desbalances entre los aportes y requerimientos de proteína y energía, lo que se vio reflejado en los metabolitos sanguíneos analizados. 


\section{REFERENCIAS}

Amer HA, HAH Salem, AA AL-Hozab. 1999. Biochemical changes in serum and milk constituents during postpartum period in Saudí Ardy goats. Small Rumin Res 34(2): 167173.

AOAC, Association of Official Analytical Chemists. 1984. Official Methods of Analysis. 14 ${ }^{\mathrm{a}}$ ed. Sidney Williams, USA.

Bed S, E Nikodemusz, Z Nagy, J Seregi. 1998. Milk urea and lactose as indicators of the protein and energy status in lactating ewes and goats. En: REU. Technical Series. Food and Agriculture Organization (FAO), Rome. 50: 204-211.

Bertoni G. 1999. Guida alla interpretazione dei profilimetabolici. Associazione Scientifica di Produzione Animale. Università Degli Studi Perugia, pp. 10-28.

Cabiddu, A, G Molle, A Branca, M Decandia, A Pes, PM Santucci, F Masoero, L Calamari. 1998. Relationship between BCS, metabolic profile and milk quantity and quality in goats browsing a mediterranean shrubland. Stocartsvo 52: 202-207.

Cannas, B, A Pes, R Mancuso, J Vodret, A Nudda. 1998. Effect of dietary energy and protein concentration on the concentration of milk urea nitrogen in dairy ewes. J Dairy Sci 81:499-508.

Contreras PA, L Valenzuela, F Wittwer y H Böhmwald. 1996. Desbalances metabólicos nutricionales más frecuentes en rebaños de pequeños productores de leche, Valdivia- Chile. Arch Med Vet 28: 39-50

Cunningham J. 1996. Fisiología Veterinaria. Interamericana, Ciudad de México: Mc Graw-Hill.

Chang CJ, CF Chen and CP Wu. 1997. Changes in apparent mammary uptake of blood metabolites during involution in dairy goats. Small Rumin Res 24 (1): 49-54.

Chiofalo V, R Maldonat, B Capogreco, G Barrico. 2000. Rumen-protect methionine and lisin in dairy goat: effect on metabolic profile tests. En: VII. International Conference on Goats. Lyon, France. pp. 92.

Chunleau I. 1994. Manuel pratique d'élevage caprin. Cap. 3. La Alimentation. Evaluation de L'état corporel. France : Ed. L'Ucarec. pp. 57-63.

Greppi GF, A Ciceri, M Pasquini, U Falasch, G Enne. 1995. Milk yield in dairy goats and blood metabolites. En: Proceedings of IDF- Seminar on Production and Utilization of Ewe's and Goat's Milk. October, Greece. pp 19-21.

Gustafsson A, J Carlsson. 1993. Effects of silage quality, protein evaluation systems and milk urea content on milk yield and reproduction on dairy cows. Livest Prod Sci 37: 91-105.

Hussain Q, O Havrevoll, LO Eik, E Ropstad. 1996. Effects of energy intake on plasma glucose, non-esterified fatty acids and acetoacetate concentration in pregnant goats. Small Rumin Res 21 (2): 89-96.

IICA, Instituto Interamericano de Cooperación para la Agricultura. 1992. Simulación de Sistemas Pecuarios. Cap. 1. Cañas, R. y Aguilar, C. (ed.) IICA, San José, Costa Rica.

ITOVIC, Institut Technique de l'Elevage Ovin et Caprin. 1982. Pratique de l'alimentation des caprins. Chap. 2. Ed. ITOVIC. París, Francia.

Khaled N, J Illek, S Gajdusek. 1999. Interactions between nutrition, blood metabolic profile and milk composition in dairy goats. Acta Vet Brno 68: 253-258.

Khaled N, J Illek. 2000. Effect of dietary supplementation of rumen- protected metionine on selected blood and milk constituents in dairy goats. En: VII International Conference on Goats. Lyon, France. pp. 89-91.

Khaled N, J Illek. 2002. Selected blood and milk indicators in healthy and ketotic goats. En: XXII World Buiatrics Congress, Hannover, Germany.

Marín MP, JC Ramos, E Fieldhouse, J Burrows. 2000. Evaluación productiva lechera en cabras sometidas a diferente sistema de explotación en la región central de Chile. En: XXI Congreso Mundial de Buiatría. Punta del Este, Uruguay.

Matthews, JG. 1999. Diseases of the Goat. $2^{\text {th }}$ edition. Iowa State University Press, Ames, Iowa.

NRC, National Research Council, USA. 1981. Nutrient Requirements of Goats: Angora, Dairy, and Meat Goats in Temperate and Tropical Countries. Washington, D.C.: Ed. National Academy Press.

Oltner R, H Wiktorsson. 1983. Urea concentrations in milk and blood as influenced by feeding varying amounts of protein and energy to dairy cows. Livest Prod Sci 10: 457468.

Ríos C, MP Marín, A Murasso, W Rudolph. 2001.Concentración de urea en sangre y leche de cabras y su correlación en sistemas intensivos lecheros de la región metropolitana. Av Cs Vet 16: 52-57.

S.A.S. / STAT. 1985. Statistical Analysis System. Guide personal computer. $6^{\text {th }}$ ed. Minnesota.

Tanwar R, A Tinna, A Gahlot, S Sharma. 2000. Biochemical profile of clinical ketosis in goats. En: VII International Conference on Goats, Lyon, France. pp. 306-310.

Wittwer F, H Opitz, J Reyes, PA Contreras y H Böhmwald. 1993. Determinación de urea en muestras de leche de rebaños bovinos para el diagnóstico de desbalance nutricional. Arch Med Vet 25: 165-172.

Wittwer F. 2000. Empleo Estratégico de Indicadores Bioquímicos en el Control de problemas metabólicos nutricionales en bovinos. En: XIII Reunión Científico Técnica. Asociación Argentina de Veterinarios de Laboratorios de Diagnóstico. Merlo, San Luis, Argentina. 